\title{
Seminare 2007
}

\section{Praxiscomputerworkshop}

\section{Inhalt}

Unter der Leitung von SGAM.Informatics erhalten die Workshopteilnehmer/innen im 1. Teil eine Einführung in die Anforderungen eines Praxisinformationssystems. Anhand einer modernen vernetzten Praxisinfrastruktur werden die Beurteilungskriterien für eine praxis- und zukunftstaugliche Softwarelösung dargestellt. Checklisten sollen die schnelle Orientierung unterstützen und bei der Beurteilung und Wahl des Produkts konkrete Hilfe bieten. Der 2. Teil umfasst die Präsentation von sechs Praxisadministrationssoftwarelösungen (Leistungserfassung, elektronisches Abrechnen unter Einbezug der TrustCenter, Agendaführung, Statistiken, Laborgeräteeinbindung, elektronische Krankengeschichte, Finanzbuchhaltungslösungen usw.).

\section{Ziel}

Die Workshopteilnehmer/innen erhalten produkteunabhängige Beurteilungskriterien für ein modernes Praxisinformationssystem. Zudem erhalten sie einen ersten Überblick über führende Softwarelösungen.

\section{Kosten}

Für FMH-Mitglieder gratis.

\section{Datum}

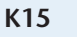

Donnerstag, 29. März 2007

Kongresshaus Zürich 9.30-16.30 Uhr

Folgende Softwareanbieter können Sie im 2. Teil des Workshops kennenlernen:

\section{Ärztekasse, Urdorf (CB 6)}

Als Marktleader seit 1964 im Bereich Praxisadministration können Sie bei der Ärztekasse auf eine ganzheitliche Lösung für Ihre Fragen betreffend Abrechnungsvarianten, Computerwahl, Netzwerke, Formular- und Briefbearbeitung usw. zählen. Die statistischen Erhebungen (im Auftrag der FMH Roko) sind ein anerkanntes betriebswirtschaftliches und standesorganisatorisches Hilfsmittel für die moderne Praxisführung. Nebst innovativen Ideen und Lösungen sowie Kooperationspartnerschaft mit 10 TrustCentern ist uns eine umfassende Kundenbetreuung ein Anliegen. Unsere Standorte befinden sich in
Basel, Bern, Chur, Crissier, Genf, Le Landeron, Lugano, Luzern, St. Gallen, Thônex und Zürich. Sie definieren Ihre Wünsche - wir bieten Ihnen die Lösung!

\section{Axon Lab AG, Dättwil (Achilles ${ }^{\mathrm{TM}}$ )}

Axon Lab AG ist ein unabhängiges, seit 15 Jahren auf dem Markt etabliertes Schweizer Unternehmen im Bereich Life Science, medizinische Labordiagnostik und Praxisadministration. Einen Fokus der Geschäftstätigkeit bildet die Zusammenarbeit mit dem Praxisarzt. Die Stärke von Axon Lab AG liegt einerseits in der Aktualität und hervorragenden Qualität des Gesamtangebots und andererseits in der Nähe und langjährigen persönlichen Beziehung zu den Kunden. Ihre spezifischen Bedürfnisse zu erkennen und sie in kundenorientierte Lösungen umzusetzen, ist die eigentliche Triebfeder für unsere Tätigkeit, Achilles $^{\mathrm{TM}}$ bietet dem Arzt die beste Möglichkeit seine Praxisadministration effizient, sicher, zukunftsorientiert und einfach in der Anwendung zu erledigen. Der modulare Aufbau von Achilles $^{\mathrm{TM}}$ sowie die zugrundeliegende Datenbank sorgen nicht nur heute, sondern auch in Zukunft für ein bedürfnisorientiertes und zeitsparendes Arbeiten. Die Software ist in drei Grundausführungen erhältlich. Neben der eigentlichen Praxisadministration in Achilles ${ }^{\mathrm{TM}}$ factura und Achilles $^{\mathrm{TM}}$ medicus ist Achilles ${ }^{\mathrm{TM}}$ lab als eigenständige Laborlösung erhältlich. Die Vernetzung der Laborgeräte mit der Software erleichtert die Abläufe im Praxislabor und erfüllt die Anforderungen an die interne Qualitätskontrolle.

InfoCall Produkte AG, Zug (MEDICOwin ${ }^{\circledR}$ ) Kompetenz, Qualität und Kontinuität sind seit mehr als 25 Jahren Leitspruch unserer Firma. Wir beraten anspruchsvolle Kunden in allen Belangen der Informationstechnologie. Unsere modular einsetzbare Software wird mit modernsten Werkzeugen nach neusten Erkenntnissen und Erfahrungen unserer Anwender entwickelt. Betriebssichere und bedienerfreundliche Gesamtlösungen garantieren so optimale Wertschöpfung und lange Lebensdauer. Mit über 3000 täglich im Einsatz stehenden Programmodulen zählen wir $\mathrm{zu}$ den erfolgreichen Softwarehäusern in der Schweiz. Mit MEDICOwin ${ }^{\circledR}$ offerieren wir als erste Firma in der Schweiz die elektronische KG mit integrierter Spracherkennung. Ebenfalls als eine der ersten wurden wir für die volle Inte- 
gration der Covercard zertifiziert. Unser Migrationsprogramm MEDICO ${ }^{\mathrm{cv}}$ erlaubt Ärztinnen und Ärzten, die heute andere Softwareprodukte einsetzen, einen problemlosen Umstieg auf MEDICOwin ${ }^{\circledR}$.

\section{Kern Concept AG, Gossau (AESKULAP)}

AESKULAP ist ein extrem schnelles Windowsprogramm, das zusammen mit einer SQL-Datenbank höchste Verarbeitungsgeschwindigkeit, Kompaktheit und schnellste Zugriffsmöglichkeiten gewährleistet. AESKULAP bietet für alle Bedürfnisse eine optimale und intelligente Lösung: Vom einfachen Abrechnungssystem mit sämtlichen Leistungserfassungsmöglichkeiten bis zur vollstrukturierten elektronischen KGFührung, die in der Praxis keine Wünsche offenlässt. Rund 200 Praxen arbeiten bereits mit der elektronischen KG-Führung. AESKULAP ist ein modular aufgebautes, anpassbares System mit einer Vielzahl von Modulen.

\section{Triamun AG, Gümligen (TriaMed)}

Triamun entwickelt und vermarktet innovative Softwarelösungen für Praxis- und Apothekenmanagement. Individuelle Beratung, die gesamte Soft- und Hardware, einen umfassenden Support und Schulung aus einer Hand. Die Produkte und Dienstleistungen auf den beiden Plattformen ASP und .NET bieten für jedes Bedürfnis die geeignete Lösung. Die Softwarelösungen auf ASP-Plattform für Apotheken und Arztpraxen basieren auf browsergestützter Technologie und wurden zusammen mit Ärzten und Apothekern entwickelt. So sind intuitiv bedienbare Managementlösungen für Apotheken und Praxen entstanden, die dem Bedürfnis nach zentralem Outsourcing Rechnung tragen. Die Softwarelösungen auf .NET-Plattform, mit seinen Zusatzmodulen für die Arztpraxis, wurde während 20 Jahren den Kundenbedürfnissen ständig angepasst, so dass sie heute als modernste lokale Client-Server-Lösung oder zentrale TerminalServer-Lösung optimal auf die Abläufe in der Arztpraxis zugeschnitten sind.

\section{vitodata AG, Ohringen b. Winterthur (vitoMed-Administrationssystem)}

Die vitodata AG besteht seit mehr als 26 Jahren. Die Unternehmung konzentriert sich auf Praxisund Kliniklösungen. Die innovative Haltung eröffnet laufend neue Einsatzgebiete - immer mit dem entsprechenden Nutzen für die Anwender. In den ersten 25 Jahren stand die Abrechnung mit der ICT im Vordergrund. Heute ist die vitodata AG auch bei der elektronischen Krankengeschichtenführung an der Spitze im Schweizer Markt. Die Marktführerschaft ist für das ganze Team der vitodata AG eine Verpflichtung, im Sinne des Investitionsschutzes für die Kunden den Fortbestand $\mathrm{zu}$ sichern und unternehmerisch und ethisch korrekt zu handeln.

\section{Anmeldung und Auskunft}

Internet www.fmhservices.ch oder FMH Consulting Services, Claudine Tannari, Burghöhe 1, 6208 Oberkirch, Tel. 04192500 77, Fax 041 9210586.

\section{PRAXIS ERPROBT}

Unsere Beratungsschwerpunkte

Praxisgründung

- Standortanalysen

- Praxisplanung und -einrichtung

- Gruppenpraxenberatung

- Evaluation von Praxisadministrationssoftware

- Rechtsberatung

Praxisführung

- Factoring - Gemeinsamer Einkauf für Ärztinnen und Ärzte

- Ärztedrucksachen

- Praxislabor

- Röntgenanlageberatung

Praxisübergabe/-übernahme

- Nachfolgeplanung und -regelung

- Unternehmenswertberechnungen/Praxisverkauf

- Praxisvermittlung

Treuhand

- Finanz- und Rechnungswesen

- Analysen

- Steuern

\section{PRAXIS ERPROBT}

Unsere Beratungsschwerpunkte

Versicherungen

- Versicherungslösungen

- Umfassende Vorsorge- und Finanzplanung

- Neutrale Vermögensverwaltung

- Optimierte Steuerplanung

Seminarangebot

- Seminare zu Themen wie Praxiseröffnung/-übernahme, Praxisübergabe, Buchhaltung/Steuern, EDV-Workshop und viele andere

Praxis- und Stellenvermittlung

- Insertionsplattform in der Schweizerischen Ärztezeitung/

Stellen-/Praxisofferten und -gesuche

- Insertionsplattform unter www.fmhjob.ch

Inkasso

- Bonitätsprüfung (online via my.inkas)

- Vorrechtliches und rechtliches Inkasso

- Verlustscheininkasso

FMH Services

Postfach 246, 6208 Oberkirch, Tel. 04192500 77, Fax 0419210586 